\title{
Research Paper: Clinico-epidemiological Analysis of Adult Patients With Acute Poisoning
}

\author{
Forough Soltaninejad ${ }^{1}$ Q, Ghazal Beigi ${ }^{2}$ Q Parastoo Golshiri $^{3}$ Q , Nastaran Eizadi-Mood $^{4 *}$ C
}

1. Department of Internal Medicine, Respiratory Research Center, School of Medicine, Isfahan University of Medical Sciences, Isfahan, Iran.

2. Department of Clinical Toxicology, School of Medicine, Isfahan University of Medical Sciences, Isfahan, Iran.

3. Department of Community \& Preventive Medicine, School of Medicine, Isfahan University of Medical Sciences, Isfahan, Iran.

4. Department of Clinical Toxicology, School of Medicine, Isfahan Clinical Toxicology Research Center, Isfahan University of Medical Sciences, Isfahan, Iran

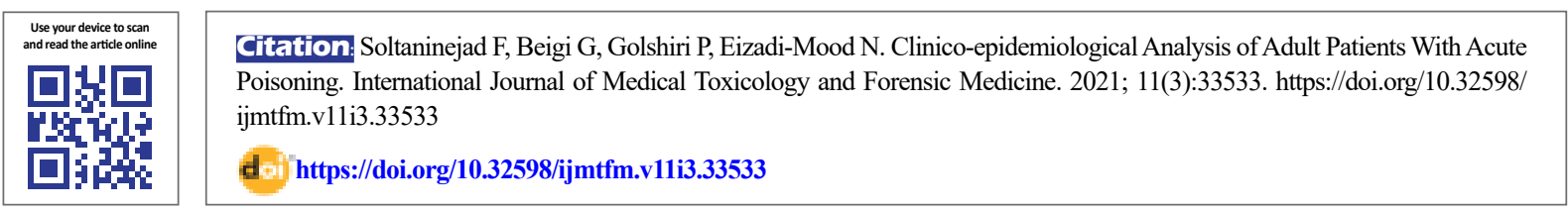

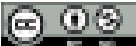

Article info:

Received: 29 Dec 2020

First Revision: 20 Jan 2021

Accepted: 31 May 2021

Published: 25 Aug 2021

\section{Keywords:}

Poisoning, Intensive care unit, Outcomes, Pesticides

\section{A B STRACT}

Background: Patients with acute poisoning sometimes require hospitalization in the Intensive Care Unit (ICU). The correct admission of these patients has resulted to lower mortality. This study compared the characteristics of adult patients with acute poisoning who were admitted to the poisoning ward and the ICU. We also determined the relevant outcome predictive factors.

Methods: This cross-sectional study was conducted on adult acutely poisoned patients who were admitted to the emergency room of Khorshid Hospital affiliated with Isfahan University of Medical Sciences from October 2018 to 2019. The clinico-epidemiological variables and outcomes were compared between adult patients hospitalized in the ward $(n=100)$ and ICU $(n=100)$. Binary logistic regression was used for predicting the outcome factors.

Results: The Mean \pm SE age of the study patients in the ICU and ward were $36.6 \pm 1.62$ (median: 34) and $34.20 \pm 1.19$ years, (median: 33, $\mathrm{P}=0.23$ ), respectively. There was no significant difference between the study groups respecting substance dependence, alcohol and drug abuse, disease history, and the type of exposure $(\mathrm{P}>0.05)$. However, self-mutilation, the kind of substance, the vital signs and level of consciousness at admission, and outcomes were significantly different between the research groups $(\mathrm{P}<0.05)$. Variables predicting outcomes (complication/death) were as follows: gender (male) (OR: 4.51; 95\%CI: 1.51-13.42; $\mathrm{P}=0.007$ ), the kind of substance (pesticides) (OR: $8.84 ; 95 \% \mathrm{CI}: 1.78-44.07 ; \mathrm{P}=0.008$ ), time to admission (OR: 1.04; 95\%CI: 1.02-1.08; $\mathrm{P}=0.002$ ), hypotension (OR: 14.88; 95\%CI: 3.01-71.58; $\mathrm{P}=0.001$ ), and the place of hospitalization (ward) (OR: 2.85; 95\%CI: 1.23-6.62; $\mathrm{P}=0.01$ ).

Conclusion: The self-mutilation, kind of substance, vital signs, level of consciousness at admission, and outcomes were significantly different between the study patients hospitalized in the ICU, compared to the ward. Gender (male), the kind of substance (pesticides), delayed arrival times, hypotension, and the place of hospitalization (ward) were essential factors for outcome (complication/death) prediction

*Corresponding Author:

Nastaran Eizadi-Mood, PhD.

Address: Department of Clinical Toxicology, Isfahan Clinical Toxicology Research Center, Isfahan University of Medical Sciences, Isfahan, Iran. Tel: +98 (313) 2222127

E-mail: izadi@med.mui.ac.ir 


\section{Introduction}

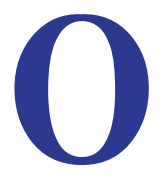

ver the last few decades, the prevalence of acute poisoning has been on the rise [1]. Poisoning, as incidentally or intentionally, is the main reason for referring to hospitals and a common route for suicide, globally. Poisoned patients are categorized at different levels respecting the need for critical care. Some patients are hospitalized in the ward while needing the Intensive Care Unit (ICU), while others are admitted to the ICU without any indication. As the mortality rate is significantly low in patients under intensive care, numerous patients with drug poisoning are transferred to the ICU just for close monitoring $[2,3]$. Concerning the limited number of ICU beds, patient categorization is vital in terms of survival [4]. The detailed evaluation of poisoned patients not only prevents unnecessary ICU admission but also increases ICU availability for the needed patients and decreases costs [5]. Therefore, without an accurate tool for the decision, we endanger the health of critical patients, waste facilities, human resources, and the increment of hospitalization costs [6].

There exist few studies on patients with drug overdose admitting to the ICU concerning the defined criteria for admission. In a published study by Banderas-Bravo on patients with drug poisoning in 3 ICUs in Spain, poisoning was a rare cause for ICU admission [7]. Lindqvist reported that $21 \%$ of poisoning cases were admitted to the ICU [8]. In the study by Van den Over, clinical assessment supplemented with Electrocardiogram (ECG) and blood gas analysis predicted the medium care/intensive care environment [9]. The results of a retrospective cohort study on adult patients with opioid poisoning in the ICU indicated that the most common indication for ICU admission was respiratory failure and the need for mechanical ventilation [10]. Athavale et al. reported that the majority of patients in poisoning ICU were due to intentional drug overdose [11]. Additionally, based on an observational study on poisoned patients in the ICU, mechanical ventilator support was required in most patients [12].

The criteria for ICU admission are dissimilar in different societies. Individuals' access to medications and toxins varies from one community to another. Moreover, the availability of pre-hospital services and hospital facili mkedso" 555555 ties conc/rning the amounts of antidote and elimination techniques is not identical. Considering the above-mentioned factors, we evaluated the clinico-epidemiological characteristics of adult patients with acute poisoning who were admitted to the ICU and wards. We also determined the outcomes of prognostic factors.

\section{Materials and Methods}

This cross-sectional study was conducted on adult acutely poisoned patients who were admitted to the emergency room of Khorshid Hospital affiliated with Isfahan University of Medical Sciences from October 2018 to 2019. This project was approved and financially supported by the Isfahan University of Medical Sciences Ethical Committee (Code: IR.MUI.MED.REC.1397.272). All patients aged $>18$ years with acute poisoning were included in the present study. Patients with envenomation and those discharged with their consent were excluded from this research. According to our local protocol, the need for endotracheal intubation due to a decreased level of consciousness, the use of mechanical ventilation, abnormal electrocardiographic, and hemodynamic changes were among the criteria for admission of patients to the ICU. The diagnosis of poisoning was based on history, clinical manifestations, and, if necessary, serological and toxicological tests and toxicology urine analysis.

The sample size was determined using the formula of comparing a variable in two communities, as follows: $95 \%$ confidence interval $(Z 1=1.96)$, test power of $80 \%$ $\mathrm{Z}(1-\mathrm{b})$ as $\mathrm{Z} 2=0.84$, and $\mathrm{P} 1=0.77$ [9], $\mathrm{P} 2=0.076$ [9], $[\mathrm{n}=200,(\mathrm{ICU}=100 \&$ ward $=100$ patients)]. The study subjects were selected by a nonprobability consecutive sampling method. Patients' data, including age, gender, the kind of used substance, time from ingestion to admission, clinical manifestations at admission, e.g. vital signs (blood pressure, temperature, respiratory rate, \& pulse rate), laboratory parameters, the duration of hospital stay, outcome (recovery without complication; complication, renal failure, hepatic failure, hypoxic encephalopathy, aspiration pneumonia, other complications, \& mortality) were recorded in a data-gathering form.

The collected data were analyzed using SPSS v. 15 . $\mathrm{P}<0.05$ was considered statistically significant. Chisquared or Fischer's Exact test and Independent Samples t-test was used in this research. The obtained results were presented as frequency (percentage), mean (standard deviation or standard error), and median. The predictive factors of outcomes were determined by binary logistic regression (backward stepwise) analysis.

\section{Results}

The study sample consisted of 200 adult patients; of whom, 100 and 100 patients were hospitalized in the ICU and ward, respectively. Moreover, $37.5 \%$ of the men and $12.5 \%$ of the women were hospitalized in the ICU $(\mathrm{P}=0.01)$. There was no gender-wise significant 
difference in age (men: $37.94 \pm 16.46 \mathrm{y}$; and in the ICU versus ward (men: $34.58 \pm 10.74 \mathrm{y}$; women: $33.65 \pm 13.50$ y) $(\mathrm{P}=0.23)$. Self-mutilation, the kind of ingested toxic agent, vital signs, and the level of consciousness at admission time were significantly different between the study groups $(\mathrm{P}<0.05)$. Comparing clinico-epidemiological variables between the study groups is presented in Table 1. Pesticide intoxication was observed in 31 patients; of them, 15 cases were aluminum phosphide poisoning and 7 cases were paraquat poisoning. Considering the kind of substance, pesticide poisoning was higher in men (23 patients; 19 in the ICU \& 4 in the ward), compared to women (8 patients; 2 in the ICU \& 6 in the ward). Additionally, opioid poisoning was more common in men, compared to women $(39.6 \%$ vs. $19.7 \%$ ), while multi-drug poisoning was more frequent in women (31.8\% vs. $21.6 \%$; $=0.002)$.

The prevalence of complications/death was higher in men, and patients poisoning with opioids and pesticides (Table 2). Binary logistic regression analysis (binary backward stepwise regression) was performed with and without adjustment for age and gender. For simplicity, recovery with complication and death cases were combined as a single ordinal variable, comprising two possible outcomes: (0) recovery without complication, (1) complication/death. The odds of complication/death in pesticide poisoning was 6.44 times higher than other poisonings (Table 3 ).

\section{Discussion}

We compared the clinico-epidemiological variables in patients with acute poisoning hospitalized in the poisoning ward and the ICU as well as the outcome predictive factors. There was no significant difference between the mean age of the explored patients hospitalized in the ICU and poisoning ward. However, as per Brandenburg [3], the mean age of ICU patients was higher, compared to the ward ones. Besides, the age of older than 55 was a predictor for treatment in the ICU.

Most of the explored patients were men, similar to other studies [13-15]. Additionally, 37.5\% of the patients hospitalized in the ICU were men. Moreover, complication/death was more in men, compared to women; thus, gender was a significant factor for outcome prediction. Similar to our result, there has been a linear relationship between increased Sequential Organ Failure Assessment (SOFA) score and male gender and adverse outcome [12]. Furthermore, male gender was correlated to in-hospital mortality in the study of Lindqvist and associates [8]. However, according to Mehrpour et al., the outcome was not significantly different between the genders; it might be due to different study protocols, as they had evaluated only patients hospitalized in the ICU [13]. The kind of substance may be another reason for gender differences in outcome, as opioids and pesticide poisoning were more common in men and multi-drug poisoning was more in women. Furthermore, there was a significant difference in the outcome of the patients respecting the kind of substances. The age of the men could not be the reason; there was no significant gender-wise difference in age concerning the place of hospitalization as well as the outcome.

Our study results indicated the mean Pulse Rate (PR) was significantly higher in ICU patients. In a study, PR of 105 has predicted patients with a need for ICU treatment [3]. Although the frequency of abnormal blood pressure was not different between patients in the ICU vs. ward, patients with hypotension presented 14.88 times higher odds of complication/death, compared to others. Some patients with abnormal hypotension were hospitalized in the ward because of the limited number of ICU beds. Brandenburg et al. reported that patients with Systolic Blood Pressure (SBP) of $<100 \mathrm{mmHg}$ required ICU treatment 3.6 times more, compared to the patients with SBP of 120-130 mmHg [3]. Abnormal blood pressure had a sensitivity of $31.4 \%$ and specificity of $92.4 \%$ in the study of Oever and colleagues [9].

The low level of consciousness was more common in our ICU patients. Studies reported that patients with Glasgow Coma Scale (GCS) of less than 6 needed ICU treatment 6.62 times more than others [3]. Moreover, Mehrpour et al. reported a GCS of less than 13 in $65 \%$ of patients in the ICU [13]. In our study, the number of patients with self-mutilation was higher in ICU than in the ward. No similar study was found in this regard. It might be due to severe psychiatric disorders which resulted in severe poisoning needed for ICU care. In a study in Turkey, a suicidal attempt was the most common cause of ICU admission in patients with poisoning [16].

In our study, the rate of complication/death was $4.5 \%$ in patients hospitalized in the ward. Complication/ death was 2.85 times higher in patients hospitalized in the ward, compared to those in the ICU. The lower resources and nurse to patients ratio in the ward and its lack of close monitoring as it is present in the ICU may explain this difference. Furthermore, some patients stay in the ward because of a limited number of ICU beds. Our results revealed the complication/death in the ICU was equal to $13 \%$. In the study about the causes of coma in patients who were admitted to poisoning ICU, $18.6 \%$ 
Table 1. Comparing different variables between the study patients hospitalized in the poisoning ward and ICU

\begin{tabular}{|c|c|c|c|c|c|}
\hline & \multirow{2}{*}{ Variables } & \multicolumn{2}{|c|}{ Groups } & \multirow{2}{*}{ Total $(\mathrm{N}=\mathbf{2 0 0})$} & \multirow{2}{*}{$\mathbf{P}$} \\
\hline & & ICU $(n=100)$ & Ward $(n=100)$ & & \\
\hline \multirow{2}{*}{ Gender } & Male & $75(37.5)$ & $59(29.5)$ & $134(67)$ & \multirow{2}{*}{0.01} \\
\hline & Female & $25(12.5)$ & $41(20.5)$ & $66(33)$ & \\
\hline History of disease & + & 27 (13.7) & $17(8.6)$ & $44(22.3)$ & 0.08 \\
\hline Smoking & + & $60(31.1)$ & $50(25.9)$ & $110(57)$ & 0.11 \\
\hline Addiction & + & $44(22.2)$ & $37(18.7)$ & 81 (40.9) & 0.31 \\
\hline Previous suicide & + & $21(10.7)$ & $20(10.2)$ & $41(20.8)$ & 0.86 \\
\hline Self-harming & + & $21(10.7)$ & $10(5.1)$ & $31(15.8)$ & 0.03 \\
\hline Drug abuser (Alcohol) & + & $4(36.4)$ & $0(0.0)$ & $4(36.4)$ & - \\
\hline \multirow{5}{*}{ Ingested toxin } & Opioids & $26(13)$ & $36(18)$ & $62(31)$ & \multirow{5}{*}{0.23} \\
\hline & Pesticides & $21(10.5)$ & $10(5)$ & $31(15.5)$ & \\
\hline & Multi drug & $25(12.5)$ & $25(12.5)$ & $50(25)$ & \\
\hline & Other medications & $15(7.5)$ & $15(7.5)$ & $30(15)$ & \\
\hline & Unknown & $13(6.7)$ & $14(7)$ & $27(13.5)$ & \\
\hline \multirow{6}{*}{ Level of consciousness } & Alert & $31(15.5)$ & $60(30)$ & $91(45.5)$ & \multirow{6}{*}{0.000} \\
\hline & Lethargic & $28(14)$ & $33(16.5)$ & $61(30.5)$ & \\
\hline & Obtundation & $5(2.5)$ & $0(0)$ & $5(2.5)$ & \\
\hline & Stupor & $16(8)$ & $4(2)$ & $20(10)$ & \\
\hline & Coma & $16(8)$ & $1(0.5)$ & $17(8.5)$ & \\
\hline & Agitation & $4(2)$ & $2(1)$ & $6(3)$ & \\
\hline $\begin{array}{l}\text { Admission pulse rate } \\
\text { (min) }\end{array}$ & Mean $\pm S D$ (median) & $94.25 \pm 22.38(89)$ & $86.98 \pm 15.82(85)$ & $90.61 \pm 19.67(86)$ & 0.009 \\
\hline \multirow{2}{*}{ Cardiac conduction } & Normal & $94(47)$ & $100(50)$ & $194(97)$ & \multirow{2}{*}{0.03} \\
\hline & Abnormal & $6(3)$ & $0(0)$ & $6(3)$ & \\
\hline \multirow{2}{*}{ Respiratory system } & Normal & $73(36.5)$ & $96(48)$ & $196(84.5)$ & \multirow{2}{*}{0.000} \\
\hline & Abnormal & $27(13.5)$ & $4(2)$ & $31(15.5)$ & \\
\hline \multirow{2}{*}{ Oxygenation } & Normal & $70(35)$ & $90(45)$ & $160(80)$ & \multirow{2}{*}{0.000} \\
\hline & Abnormal & $30(15)$ & $10(5)$ & $40(20)$ & \\
\hline \multirow{2}{*}{ Blood pressure* $^{*}$} & Normal & $93(46.5)$ & $97(48.5)$ & $190(95)$ & \multirow{2}{*}{0.33} \\
\hline & Abnormal & $7(3.5)$ & $3(1.5)$ & $10(5)$ & \\
\hline \multirow{2}{*}{\multicolumn{2}{|c|}{ Time from ingestion to admission (hours) }} & $10.91 \pm 1.60$ & $10.69 \pm 1.89$ & $10.79 \pm 1.24$ & \multirow{2}{*}{0.92} \\
\hline & & (median, 6) & (median, 5) & (median, 5) & \\
\hline \multirow{2}{*}{ Outcome } & Recovered without complication & $74(37)$ & $91(45.5)$ & $165(82.5)$ & \multirow{2}{*}{0.002} \\
\hline & Complications/ death & $26(13)$ & $9(4.5)$ & $35(17.5)$ & \\
\hline
\end{tabular}

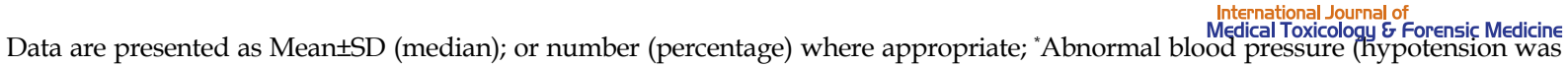
considered systolic blood pressure less than $90 \mathrm{mmHg}$ ). 
Table 2. Comparing different variables concerning outcome in the evaluated patients

\begin{tabular}{|c|c|c|c|c|c|}
\hline \multicolumn{2}{|c|}{ Variables } & $\begin{array}{l}\text { Recovered Without } \\
\text { Complications ( } n=165)\end{array}$ & $\begin{array}{l}\text { Complications/ } \\
\text { Death }(n=35)\end{array}$ & Total $(\mathrm{N}=\mathbf{2 0 0})$ & $\mathbf{P}$ \\
\hline \multirow{2}{*}{ Gender } & Male & $103(51.5)$ & $31(15.5)$ & $134(67)$ & \multirow{2}{*}{0.003} \\
\hline & Female & $62(31)$ & $4(2)$ & $66(33)$ & \\
\hline \multirow{2}{*}{\multicolumn{2}{|c|}{ Age (y) }} & $34.99 \pm 14.46$ & $37.38 \pm 12.85$ & & \multirow{2}{*}{0.37} \\
\hline & & (median; 32) & (median: 36.5) & & \\
\hline \multirow{5}{*}{ Ingested toxin } & Opioids & $49(24.6)$ & $13(6.5)$ & $62(31)$ & \multirow{5}{*}{0.003} \\
\hline & Multidrug & $43(21.5)$ & $7(3.5)$ & $50(25)$ & \\
\hline & Pesticides & $19(9.5)$ & $12(6)$ & $31(15.5)$ & \\
\hline & Others & $28(14)$ & $2(1)$ & $30(15)$ & \\
\hline & Unknown & $26(13)$ & $1(0.5)$ & $27(13.5)$ & \\
\hline \multicolumn{2}{|c|}{ Smoking } & $88(45.6)$ & $22(11.4)$ & $110(57)$ & 0.21 \\
\hline \multicolumn{2}{|c|}{ Addiction } & $62(31.3)$ & $19(9.6)$ & 81 (40.9) & 0.07 \\
\hline \multicolumn{2}{|c|}{ History of disease } & $37(18.8)$ & $7(3.6)$ & $44(22.4)$ & 0.78 \\
\hline \multirow{6}{*}{ Level of consciousness } & Alert & $74(37)$ & $17(8.5)$ & $91(45.5)$ & \multirow{6}{*}{0.50} \\
\hline & Lethargic & $54(27)$ & $7(3.5)$ & $61(30.5)$ & \\
\hline & Obtundation & $4(2)$ & $1(0.5)$ & $5(2.5)$ & \\
\hline & Stupor & $16(8)$ & $4(2)$ & $20(10)$ & \\
\hline & Coma & $12(6)$ & $5(2.5)$ & $17(8.5)$ & \\
\hline & Agitation & $5(2.5)$ & $1(0.5)$ & $6(3)$ & \\
\hline \multirow{2}{*}{ Cardiac conduction } & Normal & $160(80)$ & 34 (17) & 194 (97) & \multirow{2}{*}{0.95} \\
\hline & Abnormal & $5(2.5)$ & $1(0.5)$ & $6(3)$ & \\
\hline \multirow{2}{*}{ Respiratory system } & Normal & $141(70.5)$ & $28(14)$ & $169(84.5)$ & \multirow{2}{*}{0.42} \\
\hline & Abnormal & $24(12)$ & $7(3.5)$ & $31(15.5)$ & \\
\hline \multirow{2}{*}{ Oxygenation } & Normal & $132(66)$ & $28(14)$ & $160(80)$ & \multirow{2}{*}{0.61} \\
\hline & Abnormal & $33(16.5)$ & $7(3.5)$ & $40(20)$ & \\
\hline \multirow{2}{*}{ Blood pressure } & Normal & $161(80.5)$ & $29(14.5)$ & $190(95)$ & \multirow{2}{*}{0.002} \\
\hline & Abnormal & $4(2)$ & $6(3)$ & $10(5)$ & \\
\hline \multicolumn{2}{|c|}{$\mathrm{ICU}$} & $74(37)$ & $26(13)$ & $100(50)$ & \multirow{2}{*}{0.002} \\
\hline Wa & & $91(45.5)$ & $9(4.5)$ & $100(50)$ & \\
\hline \multirow{2}{*}{\multicolumn{2}{|c|}{ Time from ingestion to admission (hours) }} & $7.93 \pm 0.86$ & $22.96 \pm 4.71$ & $10.79 \pm 1.24$ & \multirow{2}{*}{0.000} \\
\hline & & (median: 5) & (median: 10) & (median: 5) & \\
\hline \multirow{2}{*}{\multicolumn{2}{|c|}{ Admission Pulse rate (/min) }} & $89.57 \pm 18.28$ & $95.53 \pm 24.99$ & $90.61 \pm 19.67$ & \multirow{2}{*}{0.10} \\
\hline & & (median: 86) & (median: 90) & (median: 86) & \\
\hline
\end{tabular}


Table 3. Predictive factors for outcome in the evaluated patients

\begin{tabular}{|c|c|c|c|}
\hline \multicolumn{2}{|c|}{ Variables } & \multirow{2}{*}{$\begin{array}{c}\text { OR(95\% Cl) Adjusted }{ }^{*} \\
5.82(1.64-20.65)(P=0.006)\end{array}$} & \multirow{2}{*}{$\begin{array}{l}\text { OR(95\% CI) Non Adjusted } \\
4.66(1.57-13.84)(P=0.006)\end{array}$} \\
\hline \multirow{4}{*}{ Outcome prediction } & Gender (men) $\eta$ & & \\
\hline & Ingested toxin ${ }^{*}$ (pesticides) & $6.44(1.23-33.61)(P=0.02)$ & $8.84(1.78-44.07)(P=0.008)$ \\
\hline & $\begin{array}{l}\text { Time from ingestion to admis- } \\
\operatorname{sion}^{*} \text { (Hour) }\end{array}$ & $1.04(1.02-1.08)(P=0.002)$ & $1.05(1.03-1.09)(P<0.0001)$ \\
\hline & Place of hospitalization* (Ward) & $2.85(1.23-6.62)(P=0.01)$ & $3.55(1.56-8.04)(P=0.002)$ \\
\hline \multicolumn{2}{|c|}{ Hypotension } & $14.88(3.01-71.58)(P=0.001)$ & $8.32(2.21-31.34)(P=0.002)$ \\
\hline
\end{tabular}

of patients were expired [17]. Also, the mortality rate of patients admitted to poisoning ICU was reported at $4.1 \%$ and $6.1 \%$ in other studies $[18,19]$.

Poisoning with opioids, pesticides, and multi-drugs were the most common cases in patients hospitalized in the ICU. In the study by Athavale, benzodiazepines were the most drug poisoning cases admitted to the ICU [11]. Additionally, in another study, [13] the most common causes of intoxication in the ICU admission were pharmaceutical drugs (36.6\%), opioids (26.2\%), and pesticides $(13.9 \%)$. The reason for the difference may be related to the availability of multiple medications and toxins in various communities. Pesticide poisoning was an important factor in outcome prediction in our study. Most of the patients with pesticide poisoning had ingested aluminum phosphide tablets (15 cases) and paraquat solution (7 cases). There is no definite treatment for aluminum phosphate and paraquat poisoning; thus, mortality remains high with these two pesticides [20, 21].

Finally, patients who presented late to the hospital manifested greater complications and mortality. The time between ingestion to admission has a great impact on the efficiency of treatments, especially antidotes, thus risk the outcomes [22]. Delayed arrival times were associated with higher severity scores concerning the gastrointestinal, kidney, and nervous system in toddlers [23].

\section{Conclusion}

In summary, self-mutilation, the kind of substance, vital signs, the level of consciousness at admission, and outcomes were significantly different between patients hospitalized in the ICU and ward. Gender (men), the kind of substance (pesticides), time to admission, hypotension, and the place of hospitalization (ward) were significant factors for outcome (complication/death) prediction.
Therefore, for a better therapeutic outcome in patients with acute poisoning, it might be suggested besides the major parameters (breathing abnormality, cardiac conduction disorders, oxygenation abnormality, need for mechanical ventilation, and abnormal blood pressure), some other parameters (toxic agent, e.g. pesticides, the gender of patients, time from toxic ingestion to admission) for ICU admission of patients may be considered.

\section{Ethical Considerations}

\section{Compliance with ethical guidelines}

All ethical principles are considered in this article. The participants were informed of the purpose of the research and its implementation stages. They were also assured about the confidentiality of their information and were free to leave the study whenever they wished, and if desired, the research results would be available to them. A written consent has been obtained from the subjects. principles of the Helsinki Convention was also observed. This study was approved by the Ethics Committee of the Isfahan University of Medical Sciecnes (Code: IR.MUI. MED.REC.1397.272).

\section{Funding}

The paper was extracted from the MD thesis (No. 397645).

\section{Author's contributions}

Conception and design: Nastaran Eizadi-Mood, Forogh Soltaninejad, Ghazal Beigi, Parastoo Golshiri; Data collection: Ghazal Beigi; Data analysis and interpretation: Parastoo Golshiri and Nastaran Eizadi-Mood; Preparing the original draft: Nastaran Eizadi-Mood, Forogh Soltaninejad, Ghazal Beigi, and Parastoo Golshiri. All authors approved the final version of the manuscript prior to submission. 


\section{Conflict of interest}

The authors declared no conflicts of interest.

\section{Acknowledgments}

We appreciate the collaboration of the archives staff of Khorshid Hospital.

\section{References}

[1] Martins SS, Sampson L, Cerdá M, Galea S. Worldwide Prevalence and trends in unintentional drug overdose: A systematic review of the literature. Am J Public Health. 2015; 105(11):e29-49. [DOI:10.2105/AJPH.2015.302843] [PMID] [PMCID]

[2] Brett AS, Rothschild N, Gray R, Perry M. Predicting the clinical course in intentional drug overdose: Implications for use of the intensive care unit. Arch Intern Med. 1987; 147(1):133-7. [DOI:10.1001/archinte.147.1.133] [PMID]

[3] Brandenburg R, Brinkman S, de Keizer NF, Kesecioglu J, Meulenbelt J, de Lange DW. The need for ICU admission in intoxicated patients: A prediction model. Clin Toxicol (Phila). 2017; 55(1):4-11. [DOI:10.1080/15563650.2016.1222616] [PMID]

[4] Eizadi-Mood N, Sabzghabaee AM, Khalili-Dehkordi Z. Applicability of different scoring systems in outcome prediction of patients with mixed drug poisoning-induced coma. Indian J Anaesth. 2011; 55(6):599-604. [DOI:10.4103/0019-5049.90616] [PMID] [PMCID]

[5] Bakker J, Damen J, Van Zanten ARH, Hubben JH. [Admission and discharge criteria for intensive care departments (Dutch)]. Ned Tijdschr Geneeskd. 2003; 147(3):110-5. [PMID]

[6] Ambrosius R, Vroegop M, Jansman F, Hoedemaekers C, Aarnoutse $R$, van der Wilt $G$, et al. Acute intoxication patients presenting to an emergency department in The Netherlands: Admit or not? Prospective testing of two algorithms. Emerg Med J. 2012; 29(6):467-72. [DOI:10.1136/emj.2010.106500] [PMID]

[7] Banderas-Bravo ME, Arias-Verdú MD, Macías-Guarasa I, Aguilar-Alonso E, Castillo-Lorente E, Pérez-Costillas L, et al. Patients admitted to three Spanish intensive care units for poisoning: Type of poisoning, mortality, and functioning of prognostic scores commonly used. Biomed Res Int. 2017; 2017:5261264. [DOI:10.1155/2017/5261264] [PMID] [PMCID]

[8] Lindqvist E, Edman G, Hollenberg J, Nordberg P, Ösby U, Forsberg S. Intensive care admissions due to poisoning. Acta Anaesthesiol Scand. 2017; 61(10):1296-304. [DOI:10.1111/ aas.13005] [PMID]

[9] Van den Oever HL, van Dam M, van't Riet E, Jansman FG. Clinical parameters that predict the need for medium or intensive care admission in intentional drug overdose patients: A retrospective cohort study. J Crit Care. 2017; 37:156-61. [DOI:10.1016/j.jcrc.2016.09.020] [PMID]

[10] Pfister GJ, Burkes RM, Guinn B, Steele J, Kelley RR, Wiemken TL, et al. Opioid overdose leading to intensive care unit admission: Epidemiology and outcomes. J Crit Care. 2016; 35:29-32. [DOI:10.1016/j.jcrc.2016.04.022] [PMID]

[11] Athavale V, Green C, Lim KZ, Wong C, Tiruvoipati R. Characteristics and outcomes of patients with drug overdose requiring admission to Intensive Care Unit. Australas Psychiatry. 2017; 25(5):489-93. [DOI:10.1177/1039856217706824] [PMID]

[12] Orsini J, Din N, Elahi E, Gomez A, Rajayer S, Malik R, et al. Clinical and epidemiological characteristics of patients with acute drug intoxication admitted to ICU. J Community Hosp Intern Med Perspect. 2017; 7(4):202-7. [DOI:10.1080/20009666 .2017.1356189] [PMID] [PMCID]

[13] Mehrpour O, Akbari A, Jahani F, Amirabadizadeh A, Allahyari E, Mansouri B, et al. Epidemiological and clinical profiles of acute poisoning in patients admitted to the intensive care unit in eastern Iran (2010 to 2017). BMC Emerg Med. 2018; 18(1):30. [DOI:10.1186/s12873-018-0181-6] [PMID] [PMCID]

[14] Khodabandeh F, Emamhadi MA, Mostafazadeh B. Epidemiological assessment of acute poisoning death one year survey. Int J Med Toxicol Forensic Med. 2012; 2(3):103-9. [doi:10.22037/ijmtfm.v2i3(Summer).3864]

[15] Dines AM, Wood DM, Yates C, Heyerdahl F, Hovda KE, Giraudon I, et al. Acute recreational drug and new psychoactive substance toxicity in Europe: 12 months data collection from the European Drug Emergencies Network (Euro-DEN). Clin Toxicol. 2015; 53(9):893-900. [DOI:10.3109/15563650.2015 .1088157] [PMID]

[16] Goksu S, Yildirim C, Kocoglu H, Tutak A, Oner U. Characteristics of acute adult poisoning in Gaziantep, Turkey. Toxicol Clin Toxicol. 2002; 40(7):833-7. [DOI:10.1081/CLT120016953] [PMID]

[17] Hassanian- Moghaddam H, Kolahi AA, Yaghooti N. [Evaluation causes of coma in patients who admitted in poisoning ICU in Loghman Hakim Hospital (Persian)]. J Iran Soc Anesthesiol Intensive Care. 2009; 30(63-64):11-20. https:/ /www. sid.ir/en/journal/ViewPaper.aspx?id=178319

[18] Shokrzadeh M, Hoseinpoor R, Jafari D, Delaram A, Pouyan Sadr A, Deylami M, et al. [A Ten-year study of drug poisoning cases admitted to the intensive care unit of Azar hospital in Gorgan, Iran (Persian)]. J Clin Basic Resh. 2019; 3(2):8-13. [DOI:10.29252/jcbr.3.2.8]

[19] Strom J, Thisted B, Krantz T, Sorensen MB. Self-poisoning treated in an ICU: Drug pattern, acute mortality and shortterm survival. Acta Anesthesiol Scand. 1986; 30(2):148-53. [DOI:10.1111/j.1399-6576.1986.tb02386.x] [PMID]

[20] Alnasser S, Hussain SM, Kirdi TS, Ahmed A. Aluminum phosphide poisoning in Saudi Arabia over a nine-year period. Ann Saudi Med. 2018; 38(4):277-83. [DOI:10.5144/02564947.2018.277] [PMID] [PMCID]

[21] Oghabian Z, Williams J, Mohajeri M, Nakhaee S, Shojaeepour S, Amirabadizadeh A, et al. Clinical features, treatment, prognosis, and mortality in paraquat poisonings: A hospitalbased study in Iran. J Res Pharm Pract. 2019; 8(3):129-36. [DOI:10.4103/jrpp.JRPP_18_71] [PMID] [PMCID]

[22] Dart RC, Erdman AR, Olson KR, Christianson G, Manoguerra AS, Chyka PA, et al. Acetaminophen poisoning: An evidence-based consensus guideline for outof-hospital management. Clin Toxicol. 2006; 44(1):1-18 [DOI:10.1080/15563650500394571] [PMID] 
[23] Alanazi MQ, Al-Jeraisy M, Salam M. Severity scores and their associated factors among orally poisoned toddlers: A cross sectional single poison center study. BMC Pharmacol Toxicol. 2016; 17:1. [DOI:10.1186/s40360-015-0044-7] [PMID] [PMCID] 